Algebraic 83 Geometric $\mathcal{T}_{\text {opology }}$

Volume 4 (2004) 1-22

Published: 9 January 2004

ATG

\title{
The concordance genus of knots
}

\author{
Charles Livingston
}

\begin{abstract}
In knot concordance three genera arise naturally, $g(K), g_{4}(K)$, and $g_{c}(K)$ : these are the classical genus, the 4-ball genus, and the concordance genus, defined to be the minimum genus among all knots concordant to $K$. Clearly $0 \leq g_{4}(K) \leq g_{c}(K) \leq g(K)$. Casson and Nakanishi gave examples to show that $g_{4}(K)$ need not equal $g_{c}(K)$. We begin by reviewing and extending their results.

For knots representing elements in $\mathcal{A}$, the concordance group of algebraically slice knots, the relationships between these genera are less clear. Casson and Gordon's result that $\mathcal{A}$ is nontrivial implies that $g_{4}(K)$ can be nonzero for knots in $\mathcal{A}$. Gilmer proved that $g_{4}(K)$ can be arbitrarily large for knots in $\mathcal{A}$. We will prove that there are knots $K$ in $\mathcal{A}$ with $g_{4}(K)=1$ and $g_{c}(K)$ arbitrarily large.

Finally, we tabulate $g_{c}$ for all prime knots with 10 crossings and, with two exceptions, all prime knots with fewer than 10 crossings. This requires the description of previously unnoticed concordances.
\end{abstract}

AMS Classification 57M25, 57N70

Keywords Concordance, knot concordance, genus, slice genus

\section{Introduction and basic results}

For a knot $K \subset S^{3}$, three genera arise naturally: $g(K)$, the genus of $K$, is the minimum genus among surfaces bounded by $K$ in $S^{3} ; g_{4}(K)$ is the minimum genus among surfaces bounded by $K$ in $B^{4} ; g_{c}(K)$ is the minimum value of $g\left(K^{\prime}\right)$ among all knots $K^{\prime}$ concordant to $K$. This paper investigates the relationships between these knot invariants.

The classical genus came to be fairly well understood through Schubert's work 33. proving that knot genus is additive under connected sum. The 4-ball genus is far more subtle. Even the fact that $g_{4}(K)$ can be zero for a nontrivial knot is not entirely obvious; this was seen first as a consequence of Artin's construction of a knotted $S^{2}$ in $S^{4}$ [2]. That $g_{4}(K)$ can be nonzero for a nontrivial knot 
was first proved by Fox and Milnor [9, 10] and by Murasugi, 28. The 4-ball genus remains an object of investigation; for instance, the solution of the Milnor conjecture, proved in 22], implies that for a torus knot $K, g_{4}(K)=g(K)$, in the smooth category. (This is false in the topological locally flat category, as observed by Rudolph 31.)

The concordance genus is more elusive and less studied than these two other invariants. Gordon [17, Problem 14] asked whether $g_{4}(K)=g_{c}(K)$ for all knots. Casson, in unpublished work, used the Alexander polynomial to show that the knot $6_{2}$ satisfies $g_{4}\left(6_{2}\right)=1$ and $g_{c}\left(6_{2}\right)=g\left(6_{2}\right)=2$. Independently, Nakanishi [29] used a similar argument to give examples showing that the gap between $g_{4}(K)$ and $g_{c}(K)$ can be arbitrarily large, for knots with $g_{4}$ arbitrarily large. In Section 2 we briefly review these results and give what is essentially Nakanishi's example showing that $g_{c}(K)$ can be arbitrarily large for knots with $g_{4}(K)=1$. (Obviously, if $g_{4}(K)=0$ then $g_{c}(K)=0$.) We then show that by using the signature in conjunction with the Alexander polynomial we can attain finer results: we construct knots $K$ with $g_{4}(K)=2$ and with the same Alexander polynomial as a slice knot, but with $g_{c}(K)$ arbitrarily large.

\section{Algebraic concordance and higher dimensional knot theory}

Associated to a knot $K$ and choice of Seifert surface, $F$, there is a Seifert form $V_{K}$ : this is an integral matrix satisfying $\operatorname{det}\left(V_{K}-V_{K}^{t}\right)= \pm 1$, where $V_{K}^{t}$ denotes the transpose. There is a Witt group of such Seifert forms, denoted $\mathcal{G}_{-}$, defined by Levine 23. Denoting the concordance group of knots by $\mathcal{C}_{1}$, Levine proved that the map $K \rightarrow V_{K}$ induces a homomorphism $\psi: \mathcal{C}_{1} \rightarrow \mathcal{G}_{-}$.

Knot invariants that are defined on $\mathcal{G}_{-}$are called algebraic invariants, and it is easily shown that the Alexander polynomial and signature based obstructions are algebraic. A general algebraic invariant of a knot, $g_{c}^{a}(K)$, is defined to be one half the rank of the minimal dimension representative of $V_{K}$ in $\mathcal{G}_{-}$. Everything we have discussed so far generalizes to higher dimensional concordance, where Levine proved that the map $\psi$ classifies knot concordance. Hence we have:

Theorem 1.1 In higher dimensions, $g_{c}(K)=g_{c}^{a}(K)$.

(Given a knot $K$ we can also form the hermitian matrix $(1-z) V_{K}+\left(1-z^{-1}\right) V_{K}^{t}$, over the field of fractions of $\mathbf{Q}[z], \mathbf{Q}(z)$. This induces a well defined homomorphism $\psi^{\prime}: \mathcal{C}_{1} \rightarrow W(\mathbf{Q}(z))$, where $W(\mathbf{Q}(z))$ is the Witt group of hermitian forms on vector spaces over the function field $\mathbf{Q}(z)$. There is an invariant $g_{4}^{a}(K)$ given by the minimal rank representative of the class of $\psi^{\prime}(K)$. It can be shown that $g_{4}(K) \geq g_{4}^{a}(K)$ and we conjecture that in higher dimensions this becomes an equality.) 


\section{Algebraically slice knots}

Our deepest and most subtle results concern algebraically slice knots. We begin with a definition:

Definition 1.2 The map $\psi: \mathcal{C}_{1} \rightarrow \mathcal{G}_{-}$has kernel denoted $\mathcal{A}$, the concordance group of algebraically slice knots.

Four-dimensional knot concordance is unique and especially challenging in that, unlike the higher dimensional analogs, $\mathcal{A}$ is nontrivial. In the smooth setting a number of techniques based on the work of Donaldson [8] and Witten 37] (see for example [22]) have given new insights into the structure of $\mathcal{A}$. However, in the topological locally flat category the only known obstructions to a knot in $\mathcal{A}$ being trivial are Casson-Gordon invariants [4, 5] and their extensions (for example [6]). In the language of the present paper, the results of [4, 5] can be stated as:

Theorem 1.3 There exist knots $K \in \mathcal{A}$ with $g_{4}(K) \geq 1$.

Gilmer extended this result in [15]:

Theorem 1.4 For every $N$ there exist knots $K \in \mathcal{A}$ with $g_{4}(K) \geq N$.

In the Casson-Gordon examples of twisted doubles of the unknot one has that $g_{4}(K)=g(K)=1$. In Gilmer's examples $g_{4}(K)=g(K)=N$.

Our main result concerning $\mathcal{A}$ is the following.

Theorem 1.5 For every $N$ there exists a knot $K \in \mathcal{A}$ with $g_{4}(K)=1$ and $g_{c}(K)=g(K)=N$.

To conclude this introduction we remark on the inherent challenge of proving Theorem 1.5 Showing that a given algebraically slice knot is not slice is equivalent to showing that it is not concordant to a single knot, the unknot. In the case, say, of showing that a genus 2 algebraically slice knot is not concordant to a knot of genus 1 , we have to prove that it is not concordant to any knot in an infinite family of knots, each of which is algebraically slice and hence about which one knows very little. There are of course some constraints on this family of knots based on their being genus 1, such as the Alexander polynomial, but with the added restriction that the knots are algebraically slice these do not

apply to the present problem. The main remaining tools are Casson-Gordon 
invariants, however known genus constraints based on these [13] already would apply to bound the 4-ball genus as well, so these cannot be directly applicable either. As we will see, Casson-Gordon invariants still are sufficient to provide examples, but the proof calls on two steps. The first is a delicate analysis of metabolizing subgroups for the linking forms that arise in this problem. The second is the construction of knots with Casson-Gordon invariants satisfying rigid constraints.

\section{References and conventions}

We will be working in the smooth category throughout this paper. All the results carry over to the topological locally flat category by [12.

Basic references for knot theory include [3, 30. The fundamentals of concordance and Levine's work are contained in [23, 24. The principal references for Casson-Gordon invariants are the original papers, 4, 5].

\section{Algebraic bounds on the concordance genus}

In this section we will study bounds on $g_{c}$ based on the Seifert form of the knot. All of these are easily seen to depend only on the algebraic concordance class, and hence are in fact bounds on $g_{c}^{a}(K)$. Because of this, none can yield information regarding $g_{c}(K)$ for knots $K \in \mathcal{A}$.

\subsection{Alexander polynomial based bounds on $g_{c}$}

Recall that the Alexander polynomial of a knot $K$ is defined to be $\Delta_{K}(t)=$ $\operatorname{det}\left(V_{K}-t V_{K}^{t}\right)$ where $V_{K}$ is an arbitrary Seifert matrix for $K$. It is well defined up to multiplication by $\pm t^{n}$ so we will assume that $\Delta_{K}(t) \in \mathbf{Z}[t]$ and $\Delta_{K}(0) \neq 0$. The degree of such a representative will be called the degree of the Alexander polynomial, $\operatorname{deg}\left(\Delta_{K}(t)\right)$.

A simple observation regarding the Alexander polynomial and concordance is that if a Seifert form $V$ represents 0 in $\mathcal{G}_{-}$then $\Delta_{V}(t)= \pm t^{n} f(t) f\left(t^{-1}\right)$ for some polynomial $f$ and integer $n$. (This result was mentioned in [9] and first proved in [10.) It follows that if $V_{1}$ and $V_{2}$ represent the same class in $\mathcal{G}$ then $\Delta_{V_{1}}(t) \Delta_{V_{2}}(t)= \pm t^{n} f(t) f\left(t^{-1}\right)$ for some polynomial $f$. From this we have the following basic example of Casson. 
Example 2.1 The knot $6_{2}$, illustrated in figure 1, satisfies $g_{c}\left(6_{2}\right)=2$ and $g_{4}\left(6_{2}\right)=1$. Note first that $\Delta_{6_{2}}(t)=t^{4}-3 t^{3}+3 t^{2}-3 t+1$ ([30]), an irreducible polynomial. Hence, if $6_{2}$ were concordant to a knot of genus 1 , we would then have $\Delta_{6_{2}}(t) g(t)= \pm t^{n} f(t) f\left(t^{-1}\right)$ for some polynomial $f$, integer $n$, and polynomial $g(t)$ with $\operatorname{deg}(g(t)) \leq 2$. Degree considerations show that this is impossible. On the other hand, Seifert's algorithm applied to the standard diagram of $6_{2}$ yields a Seifert surface of genus 2 .

To see that $g_{4}\left(6_{2}\right)=1$, observe that the unknotting number of $6_{2}$ is 1 (change the middle crossing) and so $6_{2}$ bounds a surface of genus 1 in the 4 -ball. It follows that $g_{4}(K) \leq 1$. On the other hand $6_{2}$ is not slice since its Alexander polynomial is irreducible, so it cannot bound a surface of genus 0 .

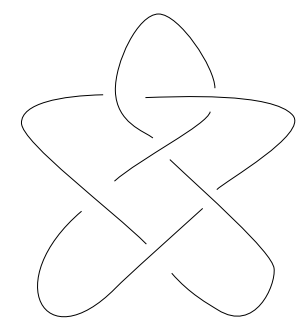

Figure 1: The knot $6_{2}$

Nakanishi [29], independently of Casson, used the Alexander polynomial in the same way to develop other examples contrasting $g_{c}$ and $g_{4}$. These techniques are summarized by the following theorem.

Theorem 2.2 Suppose that $\Delta_{K}(t)$ has an irreducible factorization in $\mathbf{Q}[t]$ as

$$
\Delta_{K}(t)=p_{1}(t)^{\epsilon_{1}} \cdots p_{k}^{\epsilon_{m}} q_{1}(t)^{\delta_{1}} \cdots q_{j}(t)^{\delta_{j}}
$$

where the $p_{i}(t)$ are distinct irreducible polynomials with $p_{i}(t)= \pm t^{n_{i}} p_{i}\left(t^{-1}\right)$ for some $n_{i}$ and $q_{i}(t) \neq \pm t^{n_{i}} q_{i}\left(t^{-1}\right)$ for any $n_{i}$. Then $g_{c}(K)$ is greater than or equal to one half the sum of the degrees of the $p_{i}$ having exponent $\epsilon_{i}$ odd.

Using this, Nakanishi proved the following. (In fact, he gives similar examples with other values of $g_{4}(K)$.) We include this argument because a related construction is used in the next subsection.

Theorem 2.3 For every $N>0$ there exists a knot $K$ with $g_{4}(K)=1$ and $g_{c}(K)>N$. 
Proof According to Kondo and Sakai, 21, 32, every Alexander polynomial occurs as the Alexander polynomial of an unknotting number one knot. Hence, the proof is completed by finding irreducible Alexander polynomials of arbitrarily high degree. Such examples include the cyclotomic polynomials $\phi_{2 p}(t)$ with $p$ an odd prime. It is well known that cyclotomic polynomials are irreducible. We have that

$$
\phi_{2 p}(t)=\frac{\left(t^{2 p}-1\right)(t-1)}{\left(t^{2}-1\right)\left(t^{p}-1\right)}=t^{p-1}-t^{p-2}+t^{p-3}-\ldots t+1 .
$$

This is an Alexander polynomial since $\phi_{2 p}(t)$ is symmetric and $\phi_{2 p}(1)=1$. Hence, the unknotting number one knot with this polynomial has $g_{4}(K)=1$ but $g_{c}(K) \geq(p-1) / 2$.

An examination of the construction used by Sakai in 32] shows that the knot used above also has $g(K)=(p-1) / 2$. Briefly, the knot is constructed from the unknot by performing +1 surgery in $S^{3}$ on an unknotted circle $T$ in the complement of the unknot $U$. The surgery circle $T$ meets a disk bounded by $U$ algebraically 0 times but geometrically $(p-1)$ times. Hence, a genus $(p-1) / 2$ surface bounded by $U$ that misses $T$ is easily constructed.

\section{$2.2 \quad$ Further bounds on $g_{c}$}

Certainly this inequality of Theorem 2.2 cannot be replaced with an equality.

Example 2.4 The granny knot (the connected sum of the trefoil with itself) has concordance genus 2 and has Alexander polynomial $\left(t^{2}-t+1\right)^{2}$. The square knot, the connected sum of the trefoil with its mirror image has the same Alexander polynomial but has concordance genus 0 . To see this, first recall that both these knots have genus 2. We have that $g_{c}(K) \geq g_{4}(K)$. According to Murasugi [28], the classical signature of a knot bounds $g_{4}$; more precisely, $g_{4}(K) \geq \frac{1}{2} \sigma(K)$. The signature of the granny knot is 4 , and hence we have the desired value of $g_{4}$ for the granny knot. On the other hand, the square knot is of the form $K \#-K$ and hence is slice.

The rest of this subsection will discuss strengthening Theorem 2.2. We begin by recalling Levine's construction of isometric structures in 23. Every Seifert form $V$ is equivalent (in $\mathcal{G}_{-}$) to a nonsingular form of no larger dimension. Associated to such a $V$ of dimension $m$ we have an isometric structure $(\langle\rangle, T$, on a rational vector space $X$ of dimension $m$, where $\langle$,$\rangle is the quadratic form on$ 
$X$ given by $V+V^{t}$ and $T$ is the linear transformation of $X$ given by $-V^{-1} V^{t}$. The map $V \rightarrow\left(V+V^{t},-V^{-1} V^{t}\right)$ defines an isomorphism from the Witt group of rational Seifert forms $\mathcal{G}^{\mathbf{Q}}$ to the Witt group of rational isometric structures, $\mathcal{G}_{\mathbf{Q}}$. The Alexander polynomial of $V$ is the characteristic polynomial of $T$. (In the Witt group $\mathcal{G}_{\mathbf{Q}}$ an isometric structure is Witt trivial, by definition, if the inner product $\langle$,$\rangle vanishes on a half-dimensional T$-invariant subspace of $X$.)

As a $\mathbf{Q}\left[t, t^{-1}\right]$ module $X$ splits as a direct sum $\oplus X_{p(t)}$ over all irreducible polynomials $p(t)$, where $X_{p(t)}$ is annihilated by some power of $p(t)$. According to Levine, any isometric structure is equivalent to one with the $X_{p(t)}$ trivial if $p(t) \neq \pm t^{n} p\left(t^{-1}\right)$ for some $n$. Furthermore, 24, Lemma 12], each remaining $X_{p(t)}$ can be reduced to a Witt equivalent form annihilated by $p(t)$ :

$$
X_{p(t)}=\left(\frac{\mathbf{Q}\left[t, t^{-1}\right]}{<p(t)>}\right)^{k}
$$

for some $k$.

Write $X$ as $\oplus_{i=1 \ldots s} X_{p_{i}}$ where the $X_{p_{i}}$ are all of the given form. Now, suppose that $p_{i}(t)$ has as a root $e^{i \theta}$ for some real $\theta$. The Milnor $\theta$-signature of $V$, $\sigma_{\theta}(V)$, (see [27]) is defined to be the signature of the quadratic form $\langle$,$\rangle re-$ stricted to the (real) summand of $X_{p_{i}(t)}$ associated to $p_{\theta}(t)=t^{2}-2 \cos (\theta) t+1$. From this analysis the next theorem follows immediately.

Theorem 2.5 Suppose $\Delta_{V}(t)$ has distinct symmetric irreducible factors $p_{i}(t)$ and $p_{i}\left(e^{i \theta_{i}}\right)=0$. If $\sigma_{\theta_{i}}(V)=2 k_{i}$ then $g_{c}^{a}(V) \geq \frac{1}{2} \sum_{i}\left|k_{i}\right|\left(\operatorname{deg}\left(p_{i}\right)\right)$.

Notice that there can be distinct values of $\theta_{i}$ for which $p_{i}\left(e^{i \theta_{i}}\right)=0$.

In general, the computation of the Milnor $\theta$-signatures can be nontrivial. The following examples illustrate how the signature used in conjunction with the Alexander polynomial yields much stronger results than can be obtained using either one alone.

Example 2.6 For a given prime $p=3 \bmod 4$, consider an unknotting number 1 knot $K$ with $\Delta_{K}(t)=\phi_{2 p}(t)$. According to Murasugi [28, if $\left|\Delta_{K}(-1)\right|=3$ $\bmod 4$ then $\sigma(K)=2 \bmod 4$, where $\sigma(K)$ is the classical knot signature, the signature of $V_{K}+V_{K}^{t}$. It is easily shown that $\phi_{2 p}(-1)=p$, so for our $K$ we have $|\sigma(K)|=2 \bmod 4$. However, since $g_{4}(K)=1,|\sigma(K)| \leq 2$. After changing orientation if need be, we have that $\sigma(K)=2$. By [26] $\sigma(K)$ is given as a sum of Milnor signatures, so it follows that for some $\theta, \sigma_{\theta}(K)=2$. Now, let $J=K \# K$. Since Milnor signatures are additive under connected 
sum, $\sigma_{\theta}(J)=4$. We also have $\Delta_{J}(t)=\left(\phi_{2 p}(t)\right)^{2}$, which is of degree $2 p-2$. Hence by the previous theorem, $g_{c}(J) \geq \operatorname{deg}\left(\phi_{2 p}(t)\right)=p-1$. No bound on $g_{c}$ can be obtained using Theorem 2.2 since the polynomial is a square. Since $J$ is unknotting number 2 , we have $c_{4}(J) \leq 2$ but the signature implies that $g_{4}(J)=2$.

\section{Casson-Gordon invariants}

\subsection{Basic theorems}

We will be working with a fixed prime number $p$ throughout the following discussion.

For a knot $K$ let $M(K)$ denote the 2-fold branched cover of $S^{3}$ branched over $K$. Let $H_{K}$ denote the $p$-primary summand of $H_{1}(M(K), \mathbf{Z})$. More formally, we have $H_{K}=H_{1}\left(M(K), \mathbf{Z}_{(p)}\right)$, where $\mathbf{Z}_{(p)}$ represents the integers localized at $p$; in other words, $\mathbf{Z}_{(p)}=\left\{\frac{m}{n} \in \mathbf{Q} \mid \operatorname{gcd}(p, n)=1\right\}$.

There is a nonsingular symmetric linking form $\beta: H_{K} \times H_{K} \rightarrow \mathbf{Q} / \mathbf{Z}$. If $K$ is algebraically slice there is a subgroup $M \subset H_{K}$ satisfying $M=M^{\perp}$ with respect to the linking form. Since the linking form is nonsingular, this easily implies that $|M|^{2}=\left|H_{K}\right|$. Such an $M$ is called a metabolizer for $H_{K}$.

Let $\chi: H_{K} \rightarrow \mathbf{Z}_{p^{k}}$ be a homomorphism. The Casson-Gordon invariant $\sigma(K, \chi)$ is a rational invariant of the pair $(K, \chi)$. (See [4, where this invariant is denoted $\sigma_{1} \tau(K, \chi)$ and $\sigma$ is used for a closely related invariant.) The main result in [CG1] concerning Casson-Gordon invariants and slice knots that we will be using is the following.

Theorem 3.1 If $K$ is slice then there is a metabolizer $M \subset H_{K}$ such that $\sigma(K, \chi)=0$ for all $\chi: H_{K} \rightarrow \mathbf{Z}_{p^{k}}$ vanishing on $M$.

We will be using Gilmer's additivity theorem [14, a vanishing result proved by Litherland [25, Corollary B2], and a simple fact that follows immediately from the definition of the Casson-Gordon invariant.

Theorem 3.2 If $\chi_{1}$ and $\chi_{2}$ are defined on $M_{K_{1}}$ and $M_{K_{2}}$, respectively, then $\sigma\left(K_{1} \# K_{2}, \chi_{1} \oplus \chi_{2}\right)=\sigma\left(K_{1}, \chi_{1}\right)+\sigma\left(K_{2}, \chi_{2}\right)$.

Theorem 3.3 If $\chi$ is the trivial character, then $\sigma(K, \chi)=0$.

Theorem 3.4 For every character $\chi, \sigma(K, \chi)=\sigma(K,-\chi)$. 


\subsection{Identifying characters with metabolizing elements}

We will be considering characters that vanish on a given metabolizer $M$. Note that the character given by linking with an element $m \in M$ is such a character and that any character $\chi: H_{K} \rightarrow \mathbf{Z}_{p^{k}}$ vanishing on $M \subset H_{K}$ is of the form $\chi(x)=\beta(x, m)$ for some $m \in M$. We will denote this character by $\chi_{m}$.

\subsection{Companionship results}

Our construction of examples of algebraically slice knots will begin with a knot $K$ with a null homologous link of $k$ components in the complement of $K$, $L=\left\{L_{1}, \ldots, L_{k}\right\} . \quad L$ will be an unlink, though it will link $K$ nontrivially. A new knot, $K^{*}$, will be formed by removing from $S^{3}$ a neighborhood of $L$ and replacing each component with the complement of a knot, $J_{i}$. This can be done in such a way that the resulting manifold is again $S^{3}$. (The attaching map should identify the meridian of $L_{i}$ with the longitude of $J_{i}$ and vice versa.) The image of $K$ in this new copy of $S^{3}$ is the knot we will denote $K^{*}$.

Let $\tilde{L}_{i}$ denote a lift of $L_{i}$ to the 2 -fold branched cover, $M(K)$. There is a natural identification of $H_{1}(M(K), \mathbf{Z})$ and $H_{1}\left(M\left(K^{*}\right), \mathbf{Z}\right)$. Suppose that $\chi: H_{K} \rightarrow \mathbf{Z}_{p^{j}}$ and that $\chi\left(\tilde{L}_{i}\right)=a_{i}$. We have the following theorem relating the associated Casson-Gordon invariants of $K$ and $K^{*}$. A proof is basically contained in [15]. The result is implicit in [25] and [14. In the formula, $\sigma_{a_{i} / p^{j}}\left(J_{i}\right)$ denotes the classical Tristram-Levine signature [36] of $J_{i}$. This signature is defined to be the signature of the hermitian form

$$
\left(1-e^{\frac{a_{i}}{p^{j}} 2 \pi i}\right) V_{J_{i}}+\left(1-e^{-\frac{a_{i}}{p^{j}} 2 \pi i}\right) V_{J_{i}}^{t} .
$$

Theorem 3.5 In the setting just described,

$$
\sigma\left(K^{*}, \chi\right)-\sigma(K, \chi)=2 \sum_{i=1}^{k} \sigma_{a_{i} / p^{j}}\left(J_{i}\right) .
$$

\section{Properties of metabolizers}

In the next section we will construct an algebraically slice knot $K$ with $g(K)=$ $N$ and $H_{K} \cong\left(\mathbf{Z}_{3}\right)^{2 N}$. We will show that it is not concordant to a knot $J$ with $g(J)<N$ by proving that if $K$ is concordant to $J$ then $\operatorname{rank}\left(H_{J}\right) \geq 2 N$. The following is our main result relating Casson-Gordon invariants and genus. With the exception of one example our applications all occur in the case of $p=3$. 
Theorem 4.1 If $K$ is an algebraically slice knot with $H_{K} \cong\left(\mathbf{Z}_{p}\right)^{2 g}$ and $K$ is concordant to a knot $J$ of genus $g^{\prime}<g$ then there is a metabolizer $M_{K} \subset H_{K}$ and a nontrivial subgroup $M_{0} \subset M_{K}$ such that for $\chi_{m}$ with $m \in M_{K}, \sigma\left(K, \chi_{m}\right)$ depends only on the class of $m$ in the quotient $M_{K} / M_{0}$. That is, if $m_{1} \in M_{K}$ and $m_{2} \in M_{K}$ with $m_{1}-m_{2} \in M_{0}$, then $\sigma\left(K, \chi_{m_{1}}\right)=\sigma\left(K, \chi_{m_{2}}\right)$.

\subsection{Metabolizers}

Theorem 4.2 If $K$ is an algebraically slice knot of genus $g$, the linking form on $H_{K}$ has a metabolizer generated by $g$ elements.

Proof Because $K$ is algebraically slice, with respect to some generating set its Seifert matrix is of the form

$$
\left(\begin{array}{ll}
0 & A \\
B & C
\end{array}\right)
$$

for some $g \times g$ matrices $A, B$, and $C$. Hence, $H_{1}(M(K), \mathbf{Z})$ has homology presented by $V_{K}+V_{K}^{t}$, which is of the form

$$
P=\left(\begin{array}{cc}
0 & D \\
D^{t} & E
\end{array}\right)
$$

for other matrices, $D$ and $E$, where $D$ has nonzero determinant. The order of $H_{1}(M(K), \mathbf{Z})$ is $\operatorname{det}(D)^{2}$.

This presentation matrix corresponds to a generating set $\left\{x_{i}, y_{i}\right\}_{i=1, \ldots, N}$. We claim that the set $\left\{y_{i}\right\}$ generates a metabolizer. First, to see that it is selfannihilating with respect to the linking form, we recall that with respect to the same generating set the linking form is given by the matrix

$$
P^{-1}=\left(\begin{array}{cc}
-\left(D^{-1}\right)^{t} E D^{-1} & \left(D^{-1}\right)^{t} \\
D^{-1} & 0
\end{array}\right) .
$$

That this is the correct inverse can be checked by direct multiplication. The lower right hand block of zeroes implies the vanishing of the linking form on $<\left\{y_{i}\right\}>$.

We next want to see that $\left\{y_{i}\right\}$ generate a subgroup of order $\operatorname{det}(D)$. Clearly the $y_{i}$ satisfy the relations given by the matrix $D$. What is not immediately clear is that the relations given by $D$ generate all the relations that the $\left\{y_{i}\right\}$ satisfy. To see this, note that any relations satisfied by the $\left\{y_{i}\right\}$ are given as a linear combination of the rows of $P$. But since the block $D$ has nonzero determinant, any such combination will involve the $\left\{x_{i}\right\}$ unless all the coefficients corresponding to the last $g$ rows of $P$ vanish. This implies that the relation comes entirely from the matrix $D$. 
Notation Suppose that the algebraically slice knot $K$ is concordant to a knot $J$. Let $M_{\#}$ be a metabolizer for $H_{K \#-J}$. Let $M_{J}$ be a metabolizer for $H_{J}$. Let $M_{K}=\left\{m \in H_{K} \mid\left(m, m^{\prime}\right) \in M_{\#}\right.$ for some $\left.m^{\prime} \in M_{J}\right\}$. For each element $m^{\prime} \in M_{J}$, set $M_{m^{\prime}}=\left\{m \in H_{K} \mid\left(m, m^{\prime}\right) \in M_{\#}\right\}$. In particular, $M_{0}=\left\{m \in H_{K} \mid(m, 0) \in M_{\#}\right\}$ and $M_{K}=\cup_{m^{\prime} \in M_{J}} M_{m^{\prime}}$. Finally, let $M_{J, 0}=$ $\left\{m^{\prime} \in H_{J} \mid\left(0, m^{\prime}\right) \in M_{\#}\right\}$.

Theorem 4.3 With the above notation, $M_{K}$ is a metabolizer for $H_{K}$.

Proof A proof of the corresponding theorem for bilinear forms on vector spaces appears in [19]. A parallel proof for finite groups and linking forms can be constructed in a relatively straightforward manner. One such proof appears in [20]. Since all metabolizers split over the $p$-primary summands, the results follow for these summands.

The set of elements $M_{0}$ is surely nonempty: it contains 0 . It is also easily seen to be a subgroup.

Lemma 4.4 If $M_{m^{\prime}}$ is nonempty then it is a coset of $M_{0}$ in $M_{K}$.

Proof The proof is straightforward. If $x, y \in M_{m^{\prime}}$ then $\left(x, m^{\prime}\right) \in M_{\#}$ and $\left(y, m^{\prime}\right) \in M_{\#}$. Hence, $(x-y, 0) \in M_{\#}$, so $x-y \in M_{0}$. Similarly, if $x \in M_{m^{\prime}}$ and $y \in M_{0}$, then $\left(x, m^{\prime}\right) \in M_{\#}$ and $(y, 0) \in M_{\#}$, so $\left(x+y, m^{\prime}\right) \in M_{\#}$ and $x+y \in M_{m^{\prime}}$.

Lemma 4.5 The map $M_{m^{\prime}} \rightarrow m^{\prime}$ induces an injective homomorphism of $M_{K} / M_{0}$ to $M_{J} / M_{J, 0}$.

Proof It must be checked that this map is well-defined. Suppose first that $M_{m^{\prime}}=M_{m^{\prime \prime}}$. Then for any $m \in M_{m^{\prime}}=M_{m^{\prime \prime}},\left(m, m^{\prime}\right) \in M_{\#}$ and $\left(m, m^{\prime \prime}\right) \in$ $M_{\#}$. Taking differences, we have that $\left(0, m^{\prime}-m^{\prime \prime}\right) \in M_{\#}$, implying that $m^{\prime}-m^{\prime \prime} \in M_{J, 0}$ as desired.

That this map is a homomorphism is trivially checked.

To check injectivity, we need to show that for all $m^{\prime} \in M_{J, 0}, M_{m^{\prime}}=M_{0}$. But $0 \in M_{m^{\prime}}$ since $\left(0, m^{\prime}\right) \in M_{\#}$ by the definition of $M_{J, 0}$. Since $0 \in M_{m^{\prime}}, M_{m^{\prime}}$ is the identity coset, as needed. 
Theorem 4.6 Let $K$ be an algebraically slice knot with $H_{K} \cong\left(\mathbf{Z}_{p}\right)^{2 g}$ and suppose that $K$ is concordant to a knot $J$ with $g(J)<g$. Then for some metabolizer $M_{\#}$ for $H_{K \#-J}$ and for any metabolizer $M_{J}$ for $H_{J}$, the subgroup $M_{0} \subset H_{K}$ is nontrivial.

Proof If $M_{0}$ is trivial we would have, by Lemma 4.5. an injection of $\left(\mathbf{Z}_{p}\right)^{g}$ into $M_{J} / M_{J, 0}$. But by Theorem 4.2 the metabolizer $M_{J}$ can be chosen so that it has rank less than $g$. It follows that a quotient will also have rank less than $g$. Hence, it cannot contain a subgroup of rank $g$.

We now prove Theorem 4.1

Theorem 4.1 If $K$ is an algebraically slice knot with $H_{K} \cong\left(\mathbf{Z}_{p}\right)^{2 g}$ and $K$ is concordant to a knot $J$ of genus $g^{\prime}<g$ then there is a metabolizer $M_{K} \subset H_{K}$ and a nontrivial subgroup $M_{0} \subset M_{K}$ such that for $\chi_{m}$ with $m \in M_{K}, \sigma\left(K, \chi_{m}\right)$ depends only on the class of $m$ in the quotient $M_{K} / M_{0}$. That is, if $m_{1} \in M_{K}$ and $m_{2} \in M_{K}$ with $m_{1}-m_{2} \in M_{0}$, then $\sigma\left(K, \chi_{m_{1}}\right)=\sigma\left(K, \chi_{m_{2}}\right)$.

Proof Since $K \#-J$ is slice, we let $M_{\#}$ be the metabolizer given by Theorem 3.1. We also have that $-J$ is algebraically slice, so we let $M_{J}$ be an arbitrary metabolizer for $H_{J}$ with $\operatorname{rank}\left(M_{J}\right)<g$ and we let $M_{K} \subset H_{K}$ be the metabolizer constructed above. We also let $M_{0}$ be the nontrivial subgroup of $M_{K}$ described above.

Let $\chi_{m_{1}}$ and $\chi_{m_{2}}$ be characters on $H_{K}$ vanishing on $M_{K}$. We are assuming further that $m_{1}$ and $m_{2}$ are in the same coset of $M_{0}: m_{1}$ and $m_{2}$ are both in $M_{m^{\prime}}$ for some $m^{\prime} \in M_{J}$. We want to show that $\sigma\left(K, \chi_{m_{1}}\right)=\sigma\left(K, \chi_{m_{2}}\right)$.

Since $m_{i} \in M_{m^{\prime}}$, we have that $\left(m_{1}, m^{\prime}\right) \in M_{\#}$ and $\left(m_{2}, m^{\prime}\right) \in M_{\#}$. Hence, by Theorem 3.1

$$
\sigma\left(K \#-J, \chi_{m_{1}} \oplus \chi_{m^{\prime}}\right)=0=\sigma\left(K \#-J, \chi_{m_{2}} \oplus \chi_{m^{\prime}}\right)
$$

The result now follows immediately from the additivity of Casson-Gordon invariants.

\section{Construction of examples}

\subsection{Description of the starting knot, $K$}

We will build a knot $K^{*}$ with the desired properties regarding $g_{c}$. The construction begins with a knot $K$ which is then modified to build $K^{*}$. In this subsection we describe $K$ and its properties. 
Figure 2 illustrates a knot $K$ and a link $L$ in its complement. The figure is drawn for the case $N=3$. The correct generalization for higher $N$ is clear. Ignore $L$ for now. The knot $K$ bounds an obvious Seifert surface $F$ of genus N. The Seifert form of $K$ is

$$
N\left(\begin{array}{ll}
0 & 1 \\
2 & 0
\end{array}\right)
$$

The homology of $F$ is generated by the symplectic basis $\left\{x_{i}, y_{i}\right\}_{i=1, \ldots, N}$. Here each $x_{i}$ is represented by the simple closed curve formed as the union of an embedded arc going over the left band of $i^{\text {th }}$ pair of bands and an embedded arc in the complement of the set of bands. The $y_{i}$ have similar representations, using the right side band of each pair.

The knot $K$ is assured to be slice by arranging that the link formed by any collection $\left\{z_{i}\right\}_{i=1, \ldots, N}$, where each $z_{i}$ is either $x_{i}$ or $y_{i}$, forms an unlink. (We are not distinguishing here between the class $x_{i}$ and the embedded curve representing the class; similarly for $y_{i}$.)

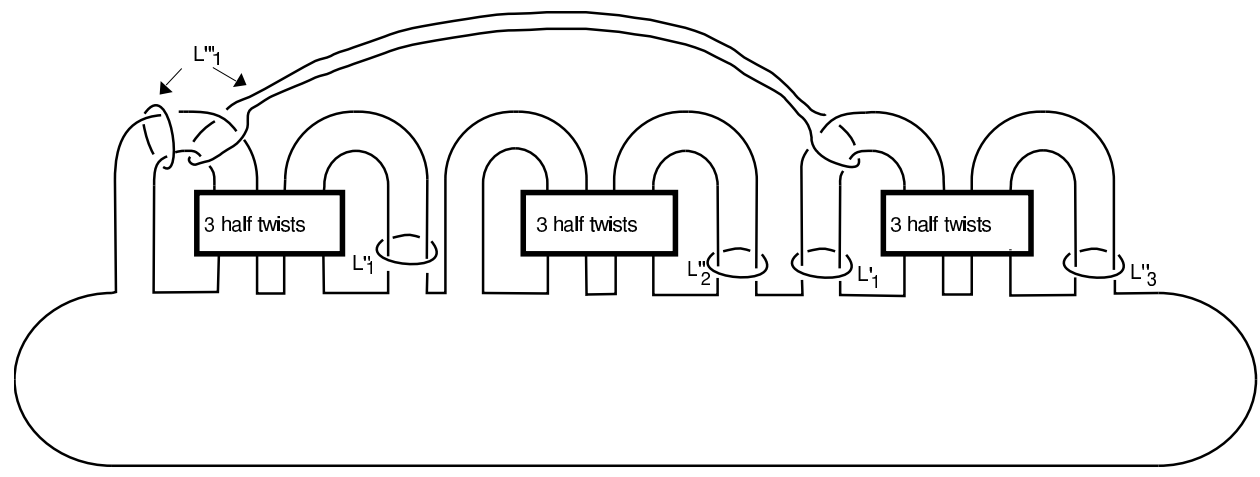

Figure 2: The basic knot

The homology of the complement of $F$ is generated by trivial linking curves to the bands, say $\left\{a_{i}, b_{i}\right\}_{i=1, \ldots, N}$. The 2 -fold cover of $S^{3}$ branched over $K, M(K)$, satisfies $H_{1}(M(K), \mathbf{Z}) \cong\left(\mathbf{Z}_{3}\right)^{2 N}$. Picking arbitrary lifts of the $\left\{a_{i}, b_{i}\right\}_{i=1, \ldots, N}$ gives a set of curves in $M(K),\left\{\tilde{a}_{i}, \tilde{b}_{i}\right\}_{i=1, \ldots, N}$, generating $H_{1}(M(K), \mathbf{Z})$. This follows from standard knot theory constructions [30, but perhaps is most evident using the surgery description of $M(K)$ given by Akbulut and Kirby [1]. It also follows easily from this description of $M(K)$ that the linking form with respect to $\left\{\tilde{a}_{i}, \tilde{b}_{i}\right\}_{i=1, \ldots, N}$ is given by

$$
N\left(\begin{array}{cc}
0 & \frac{1}{3} \\
\frac{1}{3} & 0
\end{array}\right)
$$


Here there is a slight issue of signs, but the signs as given in this linking matrix can be achieved by choosing the appropriate lifts, or simply by orienting the lifts properly.

\subsection{Construction of the link $L$}

The desired knot $K^{*}$ is constructed from $K$ by removing the components of a link $L$ in the complement of $F$ and replacing them with complements of knots $J_{i}$. In this subsection we describe $L$.

The link $L$ consists of three sublinks: $L=\left\{L^{\prime}, L^{\prime \prime}, L^{\prime \prime \prime}\right\}$. Here is how the various components of $L$ are chosen:

- $L^{\prime}$ has only one component: $L^{\prime}=\left\{L_{1}^{\prime}\right\}$. Here $L_{1}$ is chosen to be a trivial knot representing $a_{N}$, the linking circle to the band with core $x_{N}$.

- $L^{\prime \prime}=\left\{L_{i}^{\prime \prime}\right\}_{i=1, \ldots, N}$. We choose $L_{i}^{\prime \prime}$ to be a trivial knot representing $b_{i}$, the linking circle to the band with core $x_{i}$.

- $L^{\prime \prime \prime}=\left\{L_{i}^{\prime \prime \prime}\right\}$ consists of a set of 2-component sublinks. For each ordered pair, $\left(a_{i}, b_{j}\right)_{i=1, \ldots,(N-1), j=1, \ldots, N}$ we have a two component link $L_{i}^{\prime \prime \prime}$ : one component is a trivial knot representing $a_{i}$ as a small linking circle to $x_{i}$; the other component is the band connected sum of a curve parallel to that one with a trivial knot representing $b_{j}$ as a small linking circle to $b_{j}$. Similarly, 2-component links are formed for the pairs $\left(a_{i}, a_{N}\right), i<N$. The set $L^{\prime \prime \prime}$ has $N^{2}-1$ elements.

In the figure we have indicated all the components of $L^{\prime}$ and $L^{\prime \prime}$. The only sublink of $L^{\prime \prime \prime}$ that is illustrated is the one corresponding to the ordered pair $\left(a_{1}, a_{3}\right)$.

This collection is chosen so that the following theorem holds.

Theorem 5.1 A In $S^{3}-\left\{x_{i}\right\}_{i=1, \ldots,(N-1)}$ the components of $L^{\prime}$ and $L^{\prime \prime}$ form an unlink, split from the link $L^{\prime \prime \prime} \cup\left\{x_{i}\right\}_{i=1, \ldots,(N-1)}$.

B The link $L^{\prime \prime \prime} \cup\left\{x_{i}\right\}_{i=1, \ldots,(N-1)}$ is the union of an unlink, $\left\{x_{i}\right\}_{i=1, \ldots,(N-1)}$ with parallel pairs of meridians to the $x_{i}$, one pair for each sublink $L_{i}^{\prime \prime \prime}$ of $L^{\prime \prime \prime}$.

\subsection{Constructing $K^{*}$ and its properties}

We will be selecting sets of knots $\left\{J_{i}^{\prime}\right\},\left\{J_{i}^{\prime}\right\}$, and $\left\{J_{i}^{\prime \prime \prime}\right\}$. There is only one knot in the set $\left\{J_{i}^{\prime}\right\}$; it corresponds to the knot $L_{1}^{\prime}$. There are $N$ knots in 
the set $\left\{J_{i}^{\prime \prime}\right\}$, with one knot $J_{i}^{\prime \prime}$ for each $L_{i}^{\prime \prime}$. Finally, there is one knot $J_{i}^{\prime \prime \prime}$ for each 2-component sublink $L_{i}^{\prime \prime \prime}$ of $L^{\prime \prime \prime}$. The necessary properties of all these knots will be developed later. To construct $K^{*}$ we follow the companionship construction described in Section 3.3. remove tubular neighborhoods of each $L_{i}^{\prime}$ and $L_{i}^{\prime \prime}$ and replace them with the complement of the corresponding $J_{i}^{\prime}$ or $J_{i}^{\prime \prime}$. Neighborhoods of the two components of $L_{i}^{\prime \prime \prime}$ are replaced with the complements of the corresponding $J_{i}^{\prime \prime \prime}$ and its mirror image, $-J_{i}^{\prime \prime \prime}$.

Since $K^{*}$ is formed by removing copies of $S^{1} \times B^{2}$ from $S^{3}$ and replacing them with three manifolds with the same homology, the Seifert form of $K^{*}$ is the same as that of $K$. Hence, as for the knot $K, H_{1}\left(M\left(K^{*}\right), \mathbf{Z}\right) \cong\left(\mathbf{Z}_{3}\right)^{2 N}$ is presented by

$$
N\left(\begin{array}{ll}
0 & 3 \\
3 & 0
\end{array}\right)
$$

Similarly, the linking form with respect to the same basis is presented by the inverse of this matrix,

$$
N\left(\begin{array}{cc}
0 & \frac{1}{3} \\
\frac{1}{3} & 0
\end{array}\right)
$$

For framed link diagrams of these spaces, see [1].

\subsection{The concordance genus of $K^{*}$}

Before proving that the concordance genus of $K^{*}$ is $N$, we observe the following.

Theorem 5.2 The knot $K$ just constructed has $g(K)=N$ and $g_{4}(K)=1$.

Proof It is clear that $g(K) \leq N$. However, since the rank of $H_{1}(M(K), \mathbf{Z})$ is $2 N, g(K) \geq N$.

We must now show that $g_{4}(K)=1$. This is based on the observation that the curves $\left\{x_{i}\right\}_{i=1 \ldots, N-1}$ form a strongly slice link: That is, they bound disjoint disks in $B^{4}$. To see this, note that by replacing the components of the $L_{i}^{\prime \prime \prime}$ with copies of the complements of $J_{i}^{\prime \prime \prime}$ and $-J_{i}^{\prime \prime \prime}$, we have arranged that the $x_{i}$ have become the connected sums of pairs of the form $J_{i}^{\prime \prime \prime} \#-J_{i}^{\prime \prime \prime}$, and such a connected sum is a slice knot.

To build a genus 1 surface in the 4-ball bounded by $K$, simply surger the Seifert surface using these slicing disks. 
Since $H_{1}\left(M\left(K^{*}\right), \mathbf{Z}\right) \cong\left(\mathbf{Z}_{3}\right)^{2 N}$, we have, in the notation of Section 4.1, $M_{K^{*}} \cong$ $\left(\mathbf{Z}_{3}\right)^{N}$. We will be assuming that $K^{*}$ is concordant to a knot of lower genus, so we also have $M_{0}$ is a nontrivial subgroup of $M_{K^{*}}$, by Theorem 4.1. The proof that $g_{c}\left(K^{*}\right)=N$ will consist of showing that the knots $J_{i}^{\prime}, J_{i}^{\prime \prime}$ and $J_{i}^{\prime \prime \prime}$ can be chosen so that the Casson-Gordon invariants cannot be constant on the cosets of $M_{0}$.

The following result is a consequence of Theorem 3.5. Notice that there is only one term in the first sum since the link $L^{\prime}$ has just one component, which links $a_{N}$.

Theorem $5.3 \sigma\left(K^{*}, \chi\right)=\sigma(K, \chi)+2 \sum_{i} c_{i} \sigma_{1 / 3}\left(J_{i}^{\prime}\right)+2 \sum_{i} d_{i} \sigma_{1 / 3}\left(J_{i}^{\prime \prime}\right)+$ $2 \sum_{i}\left(e_{i}-e_{i}^{\prime}\right) \sigma_{1 / 3}\left(J_{i}^{\prime \prime \prime}\right)$, where:

(1) $c_{i}$ is 0 or 1 depending on whether $\chi\left(\tilde{a}_{N}\right)$ is 0 or not.

(2) $d_{i}$ is 0 or 1 depending on whether $\chi\left(\tilde{b}_{i}\right)$ is 0 or not.

(3) The values of the $e_{i}$ and $e_{i}^{\prime}$ are determined as follows. The element $L_{i}^{\prime \prime \prime} \in L^{\prime \prime \prime}$ corresponds to the class of the form $a_{k}+x \in H_{1}\left(S^{3}-F, \mathbf{Z}\right)$, where $1 \leq k \leq N-1$ and either $x=b_{l}, 1 \leq l \leq N$, or $x=a_{N}$. With this, $e_{i}$ is 0 or 1 depending on whether $\chi\left(\tilde{a}_{k}\right)$ is 0 or not; $e_{i}^{\prime}$ is 0 or 1 depending on whether $\chi\left(\widetilde{a_{k}+x}\right)$ is 0 or not.

Notation If the character $\chi$ is given by linking with an element $m \in H_{K^{*}}$, (that is, if $\chi=\chi_{m}$ ), then the coefficients $c_{i}, d_{i}$, and $e_{i}-e_{i}^{\prime}$ are functions of $m$. We denote these functions by $C_{i}, D_{i}$, and $E_{i}=e_{i}-e_{i}^{\prime}$.

Theorem 5.4 The knots $J_{i}^{\prime}, J_{i}^{\prime \prime}$, and $J_{i}^{\prime \prime \prime}$ can be chosen so that $\sigma\left(K^{*}, \chi_{m_{1}}\right)=$ $\sigma\left(K^{*}, \chi_{m_{2}}\right)$ if and only if the functions $C_{i}, D_{i}$, and $E_{i}$ all agree on $m_{1}$ and $m_{2}$.

Proof The difference $\sigma\left(K^{*}, \chi_{m_{1}}\right)-\sigma\left(K^{*}, \chi_{m_{2}}\right)$ is given by:

$$
\begin{aligned}
\sigma\left(K, \chi_{m_{1}}\right)-\sigma\left(K, \chi_{m_{2}}\right) & +2 \sum\left(C_{i}\left(m_{1}\right)-C_{i}\left(m_{2}\right)\right) \sigma_{1 / 3}\left(J_{i}^{\prime}\right) \\
& \left.+2 \sum\left(D_{i}\left(m_{1}\right)-D_{i}\left(m_{2}\right)\right)\right) \sigma_{1 / 3}\left(J_{i}^{\prime \prime}\right) \\
& +2 \sum\left(E_{i}\left(m_{1}\right)-E_{i}\left(m_{2}\right)\right) \sigma_{1 / 3}\left(J_{i}^{\prime \prime \prime}\right)
\end{aligned}
$$

The set of values of $\left\{\left|\sigma\left(K, \chi_{x}\right)-\sigma\left(K, \chi_{y}\right)\right|\right\}_{x, y \in H_{K}}$ is a finite set, so is bounded above by a constant $B$. Pick $J_{1}^{\prime}$ so that $\sigma_{1 / 3}\left(J_{1}^{\prime}\right)>2 B$. Pick $J_{1}^{\prime \prime}$ so that $\sigma_{1 / 3}\left(J_{i}^{\prime \prime}\right)>2 \sigma_{1 / 3}\left(J_{1}^{\prime}\right)$. Finally pick each following $J_{i}^{\prime \prime}$ and $J_{i}^{\prime \prime \prime}$ so that at each step the $1 / 3$ signature has at least doubled over the previous choice. 
With this choice of knots the claim follows quickly from an elementary arithmetic argument.

We will now assume that the knot $K^{*}$ has been constructed using such collections, $\left\{J_{i}^{\prime}\right\},\left\{J_{i}^{\prime}\right\}$, and $\left\{J_{i}^{\prime \prime \prime}\right\}$ as given in the previous theorem.

Lemma 5.5 The subgroup $M_{0}$ must be contained in the subgroup generated by $\left\{\tilde{b}_{i}\right\}_{i=1, \ldots, N-1}$.

Proof Consider a $\chi_{m}$ with $m \in M_{0}$. Write $m$ as a linear combination of the $\tilde{a}_{i}$ and $\tilde{b}_{i}$. If $\tilde{b}_{N}$ or some $\tilde{a}_{i}$ has a nonzero coefficient, then $\chi_{m}$ will link nontrivially with $\tilde{a}_{N}$ or some $\tilde{b}_{i}$. In this case, either $C_{1}(m)$ or some $D_{i}(m)$ will be nontrivial. (Recall that the $\tilde{a}_{i}$ and $\tilde{b}_{i}$ are duals with respect to the linking form.)

The proof of Theorem 1.5 concludes with the following.

Theorem 5.6 It is not possible for $\sigma\left(K, \chi_{m}\right)$ to be constant on each coset of $M_{0}$.

Proof To prove this, we have seen that we just need to show that one of the coefficients, either a $C_{i}, D_{i}$ or $E_{i}$, is nonconstant on some coset. In the previous proof we used the $C_{i}$ and $D_{i}$. We now focus on the $E_{i}$.

Using the previous lemma, without loss of generality we can assume that $M_{0}$ contains an element $m_{0}=\tilde{b}_{1}+\sum_{i=2, \ldots, N-1} r_{i} \tilde{b}_{i}$ for some set of coefficients $r_{i}$.

The metabolizer $M_{K}$ is of order $3^{N}$, so it must contain an element not in the span of $\left\{\tilde{b}_{i}\right\}_{i=1 \ldots, N-1}$. Adding a multiple of $m_{0}$ if need be, we can hence assume that $M_{K}$ contains an element $m=\tilde{b}_{1}+\sum_{i=2, \ldots, N-1} \beta_{i} \tilde{b}_{i}+\sum_{i=1, \ldots, N} \gamma_{i} \tilde{a}_{i}+\delta_{N} \tilde{b}_{N}$, with some $\gamma_{i}$ or $\delta_{N}$ nonzero. In fact, by changing sign, and adding a multiple of $m_{0}$, we can assume that one of the nonzero coefficients is 1 .

We can now select an element from the set $\left\{\tilde{b}_{1}, \ldots, \tilde{b}_{n}, \tilde{a}_{N}\right\}$ on which $\chi_{m}$ evaluates to be 1 . Denote that element $\tilde{b}$.

We consider the $L_{i}^{\prime \prime \prime}$ representing the pair $\tilde{a}_{1}$ and $\tilde{a}_{1}+\tilde{b}$. In this case we have the following:

- $\chi_{m}\left(\tilde{a}_{1}\right)=1$

- $\chi_{m}\left(\tilde{a}_{1}+\tilde{b}\right)=2$ 
- $\chi_{m-m_{0}}\left(\tilde{a}_{1}\right)=0$

- $\chi_{m-m_{0}}\left(\tilde{a}_{1}+\tilde{b}\right)=1$

Using Theorem 5.3 we have, for the corresponding $e_{i}$ and $e_{i}^{\prime}$ that:

- $e_{i}\left(\chi_{m}\right)\left(\tilde{a}_{1}\right)=1$

- $e_{i}^{\prime}\left(\chi_{m}\right)\left(\tilde{a}_{1}+\tilde{b}\right)=1$

- $e_{i}\left(\chi_{m-m_{0}}\right)\left(\tilde{a}_{1}\right)=0$

- $e_{i}^{\prime}\left(\chi_{m-m_{0}}\right)\left(\tilde{a}_{1}+\tilde{b}\right)=1$

Finally, from the definition of $E_{i}=e_{i}-e_{i}^{\prime}$ we have that $E_{i}\left(\chi_{m}\right)=0$ and $E_{i}\left(\chi_{m-m_{0}}\right)=-1$. Hence, the Casson-Gordon invariants cannot be constant on the coset and the proof is complete.

\section{Enumeration}

We conclude by tabulating the concordance genus for all prime knots with 10 crossings. We are also able to compute the concordance genus of all prime knots with fewer than 10 crossings with two exceptions, the knots $8_{18}$ and $9_{40}$. This is the first such listing.

In doing this enumeration we have used the knot tables contained in [3] and especially the listings of various genera in [18. Results on concordances between low crossing number knots were first compiled by Conway [7, and we have also used corrections and explications for Conway's results taken from [35]. In addition, Conway apparently failed to identify three such concordances- $-10_{103}$ and $10_{106}$ are both concordant to the trefoil, $10_{67}$ is concordant to the knot $5_{2}$ - and those concordances are described below. We also use the fact that for all knots $K$ with 10 or fewer crossings, the genus of $K$ is given by half the degree of the Alexander polynomial.

Summary In brief, there are 250 prime knots with crossing number less than or equal to 10. Of these, 21 are slice, and hence $g_{c}=0$. For 210 of them the Alexander polynomial obstruction yields a bound equal to the genus, and hence for these $g_{c}=g$. There are 17 of the remaining knots which are concordant to lower genus knots for which $g_{c}$ is known. Finally, there are two knots $8_{18}$ and $9_{40}$, for which $g_{3}=3$ but for which we have not been able to show that $g_{c}=3$. In the smooth category both of these have $g_{4}=2$ (see for instance the table in 34]) and in the topological category $g_{4}$ seems to be unknown for both, being either 1 or 2 . 


\subsection{Slice knots}

Among prime knots of 10 or few crossings there are 21 slice knots. These are $6_{1}, 8_{8}, 8_{9}, 8_{20}, 9_{27}, 9_{41}, 9_{46}, 10_{3}, 10_{22}, 10_{35}, 10_{42}, 10_{48}, 10_{75}, 10_{87}, 10_{99}$, $10_{123}, 10_{129}, 10_{137}, 10_{140}, 10_{153}$, and $10_{155}$.

\subsection{Examples for which the polynomial condition suffices}

For 210 of the 250 prime knots of 10 or fewer crossings, Theorem 2.2 gives a bound for $g_{c}(K)$ which is equal to $g(K)$. Hence for all these knots we have $g_{c}(K)=\operatorname{deg}\left(\Delta_{K}(t)\right) / 2$. This leaves 19 knots. These are:

- $8_{10}, 8_{11}, 8_{18}$

- $9_{24}, 9_{37}, 9_{40}$

- $10_{21}, 10_{40}, 10_{59}, 10_{62}, 10_{65}, 10_{67}, 10_{74}, 10_{77}, 10_{98}, 10_{103}, 10_{106}, 10_{143}, 10_{147}$

In the next subsection we will observe that 17 of these are concordant to lower genus knots with $g_{c}$ known. We have been unable to resolve the cases of $8_{18}$ and $9_{40}$.

\subsection{Concordances to lower genus knots}

Concordant to trefoil The following 8 knots are concordant to the trefoil, $3_{1}: 8_{10}, 8_{11}, 10_{40}, 10_{59}, 10_{103}, 10_{106}, 10_{143}, 10_{147}$.

As noted in [35], in [7] a typographical error leads to the knot $10_{143}$ failing to be on this list, and $10_{65}$ is placed on the list accidentally. The knots $10_{103}$, $10_{106}$ fail to be identified in [7. We will describe these concordances below. For all of these knots, $g_{c}(K)=1=g_{4}(K)$.

Concordant to the figure eight The following 2 knots are concordant to the figure eight knot, $4_{1}: 9_{24}, 9_{37}$. Both have $g_{c}(K)=1=g_{4}(K)$.

Concordant to $5_{1}$ The following 2 knots are concordant to the knot $5_{1}$ : $10_{21}, 10_{62}$. Both have $g_{c}(K)=1=g_{4}(K)$.

Concordant to $5_{2}$ The following 4 knots are concordant to the knot $5_{2}: 10_{65}$, $10_{67}, 10_{74}, 10_{77}$. The knot $10_{67}$ fails to be on the list in [7. Its concordance is described below. All have $g_{c}(K)=1=g_{4}(K)$.

Concordant to $3_{1} \# 3_{1}$ The following 1 knot is concordant to the connected sum of the trefoil with itself, $3_{1} \# 3_{1}: 10_{98}$. It satisfies $g_{c}\left(10_{98}\right)=2=g_{4}\left(10_{98}\right)$. 


\subsection{Building the concordances for $10_{67}, 10_{103}$ and $10_{106}$}

In this final subsection we will describe the concordances that were not included in Conway's list. In Figure 3 we have illustrated the knot $10_{67}$ along with a band. Performing the band move $10_{67}$ along that band results in a split link of two components: an unknot and the knot $5_{2}$. This gives the desired concordance from $10_{67}$ and $5_{2}$.

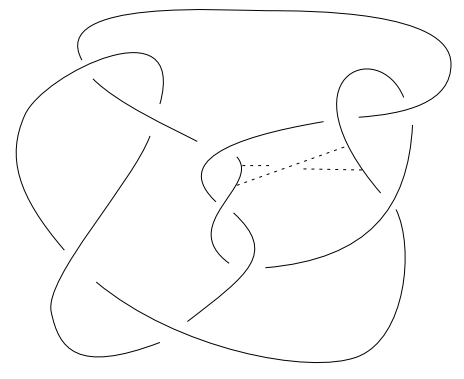

Figure 3: $10_{67}$

In Figure 4 we have illustrated the knots $10_{103} \#-3_{1}$ and $10_{106} \# 3_{1}$ along with a band in each figure. Performing the band move along each band yields a split link of two unknotted components. Hence, both $10_{103} \#-3_{1}$ and $10_{106} \# 3_{1}$ are slice, so $10_{103}$ and $10_{106}$ are each concordant to trefoil knots.

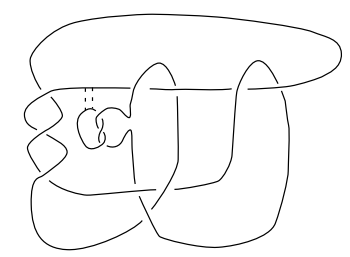

Figure 4: $10_{103} \# 3_{1}$

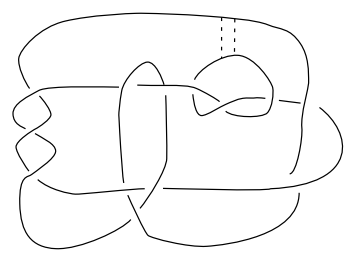

and

$$
10_{106} \# 3_{1}
$$

\section{References}

[1] S Akbulut and R Kirby, Branched covers of surfaces in 4-manifolds, Math. Ann. 252 (1979/80), 111-131

[2] E Artin, Zur Isotopie zweidimensionalen Flächen im $R_{4}$, Abh. Math. Sem. Univ. Hamburg 4 (1926), 174-177

[3] G Burde and $\mathbf{H}$ Zieschang, Knots, de Gruyter Studies in Mathematics, 5. Walter de Gruyter \& Co., Berlin-New York, 1985 
[4] A Casson and C Gordon, Cobordism of classical knots, in A la recherche de la Topologie perdue, ed. by Guillou and Marin, Progress in Mathematics, Volume 62, 1986 (Originally published as Orsay Preprint, 1975.)

[5] A Casson and C Gordon, On slice knots dimension three, Algebraic and geometric topology (Proc. Sympos. Pure Math., Stanford Univ., Stanford, Calif., 1976), Part 2, 39-53, Proc. Sympos. Pure Math., XXXII, Amer. Math. Soc., Providence, R.I., 1978

[6] T Cochran, K Orr, and $\mathbf{P}$ Teichnor, Knot Concordance, Whitney Towers and $L^{2}$ Signatures, Ann. of Math. (2) 157 (2003), 433-519

[7] J H Conway, An enumeration of knots and links, and some of their algebraic properties, Computational Problems in Abstract Algebra (Proc. Conf., Oxford, 1967), 329-358, Pergamon, Oxford, 1970

[8] S Donaldson, An application of gauge theory to four-dimensional topology, J. Differential Geom. 18 (1983), 279-315

[9] R Fox, A quick trip through knot theory, Topology of 3-Manifolds, ed. by M. K. Fort, Prentice Hall (1962), 120-167

[10] R Fox and J Milnor, Singularities of 2-spheres in 4-space and cobordism of knots, Osaka J. Math. 3 (1966) 257-267

[11] M Freedman, The topology of four-dimensional manifolds, J. Differential Geom. 17 (1982), 357-453

[12] M Freedman and F Quinn, Topology of 4-manifolds. Princeton Mathematical Series, 39, Princeton University Press, Princeton, NJ, 1990

[13] P Gilmer, On the slice genus of knots, Invent. Math. 66 (1982), 191-197

[14] P Gilmer, Slice knots in $S^{3}$, Quart. J. Math. Oxford Ser. (2) 34 (1983), 305322

[15] P Gilmer and C Livingston, The Casson-Gordon invariant and link concordance, Topology 31 (1992), 475-492

[16] C McA Gordon, Some aspects of classical knot theory, Knot theory (Proc. Sem., Plans-sur-Bex, 1977), Lecture Notes in Math., 685, Springer, Berlin, 1978, $1-60$

[17] C McA Gordon, Problems, Knot theory (Proc. Sem., Plans-sur-Bex, 1977), Lecture Notes in Math., 685, Springer, Berlin, 1978, 309-311

[18] A Kawauchi, A Survey of Knot Theory, Birkhauser Verlag, Basel, 1996

[19] M Kervaire, Knot cobordism in codimension two, Manifolds-Amsterdam 1970, (Proc. Nuffic Summer School), Lecture Notes in Mathematics, Vol. 197 Springer, Berlin (1971), 83-105

[20] P Kirk and C Livingston, Concordance and mutation, Geom. Topol. 5 (2001), 831-883

[21] H Kondo, Knots of unknotting number 1 and their Alexander polynomials, Osaka J. Math. 16 (1979), 551-559 
[22] $\mathbf{P}$ Kronheimer and T Mrowka, Gauge theory for embedded surfaces. I., Topology 32 (1993) 773-826

[23] J Levine, Knot cobordism groups in codimension two, Comment. Math. Helv. 44 (1969), 229-244

[24] J Levine, Invariants of knot cobordism, Invent. Math. 8 (1969), 98-110

[25] R Litherland, Cobordism of satellite knots, Four-Manifold Theory, Contemporary Mathematics, eds. C. Gordon and R. Kirby, American Mathematical Society, Providence RI 1984, 327-362

[26] T Matumoto, On the signature invariants of a non-singular complex sesquilinear form, J. Math. Soc. Japan 29 (1977), 67-71

[27] J Milnor, Infinite Cyclic Coverings, Topology of Manifolds, Complementary Series in Mathematics vol. 13, ed. J. G. Hocking, Prindle, Weber \& Schmidt. Boston, 1968

[28] K Murasugi, On a certain numerical invariant of link types, Trans. Amer. Math. Soc. 117 (1965), 387-422

[29] Y Nakanishi, A note on unknotting number, Math. Sem. Notes Kobe Univ. 9 (1981), 99-108

[30] D Rolfsen, Knots and Links, Publish or Perish, Berkeley CA (1976)

[31] L Rudolph, Some topologically locally-flat surfaces in the complex projective plane, Comment. Math. Helv. 59 (1984), 592-599

[32] T Sakai, A remark on the Alexander polynomials of knots, Math. Sem. Notes Kobe Univ. 5 (1977), 451-456

[33] H Schubert, Die eindeutige Zerlegbarkeit eines Knotens in Primknoten, S.-B. Heidelberger Akad. Wiss. Math.-Nat. Kl. (1949) 57-104

[34] T Shibuya, Local moves and 4-genus of knots. Mem. Osaka Inst. Tech. Ser. A 45 (2000), 1-10

[35] A Tamulis, Concordance of Classical Knots, Thesis, Indiana University, Bloomington (1999)

[36] A Tristram, Some cobordism invariants for links, Proc. Camb. Phil. Soc. 66 (1969), 251-264

[37] E Witten, Monopoles and four-manifolds, Math. Res. Lett. 1 (1994), 769-796

Department of Mathematics, Indiana University

Bloomington, IN 47405, USA

Email: livingst@indiana.edu

Received: 27 July 2003 Revised: 3 January 2004 